\title{
The Application Analysis of GPS/GIS/DMS in Xinyang Transmission Line Management
}

\author{
MingYan ${ }^{1, a}$ Juan $\mathrm{Xu}^{2, \mathrm{~b}}$ Lei Zhang $^{3, \mathrm{c}}$ \\ ${ }^{1}$ Operation and Maintenance Repair Department of Xinyang Power Company of Henan Province, \\ Xinyang, Henan, China, 464000 \\ a email, ${ }^{a}$ email, ${ }^{a}$ email,
}

Keywords: Transmission Line, GIS System, GPS Inspection, DMS Integration

\begin{abstract}
This article introduced the applications summary and exploitation experience of Xinyang Power-supplied Company Line Work Area Transmission Line GPS, did an overview of the power GPS、GIS and DMS's integration, and brought forward the new ideas of GPS and GIS's application in power field.
\end{abstract}

\section{Introduction}

GPS is the Global Positioning System. A high-precision, all-weather navigation and global positioning radio, multifunction system's timing. It uses more than 20,000 kilometers from Earth is located high by 24 satellite network consisting of satellites, the Earth is constantly transmitting positioning signals. Any GPS receiver on the earth, as long as the received signals from four or more satellites, and instantly can be calculated by the measured motion vector, such as: longitude, latitude, altitude, time, speed, heading, etc.

GIS geographic information processing system (Geophysics Information System) for the acquisition, storage, display, query positioning data and set up a computer database management system, the information and materials needed to directly and vividly on the electronic map graphics or tables form is displayed, the GPS satellite positioning to provide a good map of the environment, and can process spatial information and attribute information of the perfect combination, a visual way to display the location status.

Transmission lines, GPS is used in line inspection management, via GPS, to determine who patrol tower location and arrival time to realize the computer management line inspection work. Through a variety of data to the centralized processing line inspection, the quality of maintenance work can be tracked, so as to realize the entire power line operation and maintenance data management, improve efficiency in order to facilitate and strengthen the responsibilities of maintenance personnel, the timely detection of power line problems and risks, prevent and eliminate a major power line accidents, ensure the safe and stable operation of power lines. The use of GPS in the transmission line management is an important means of power line inspection of the implementation of modern management and it is an important step to promote the scientific management of power line maintenance.

\section{The Integrated Management System Design Program of GPS / GIS / DMS}

System design principles is to do GIS / GPS / DMS systems underlying data integration design, integration and distribution function interface integration. Its design idea is mainly to ensure the spatial database, independent and uniqueness of the historical database and real-time database. Clients' graphic definition and management is implemented on a GIS platform. Through integrated design, seamless connectivity directly to geographic information and real-time transmission of information, give full play to the performance of the system to meet the functional requirements, greatly improve the cost of the entire system.

System follows the principle of unified planning step by step, the establishment of power line 
towers GIS applications and build a complete GIS environment, including GIS data storage, display GIS to meet the demand, while the system can smooth upgrade of horizontal and vertical extensions.

It needs to establish an independent power line tower information database which includes towers attribute information and spatial information database to achieve unified management.

Standard, open, scalable interface, in case there is a demand to make other business systems can access the system data space. When defining the outer boundary of the system as much as possible the use of international agreements on open standards or technology, able to integrate other business systems integrated into the GIS system.

The system uses B / S structure, the user through the client browser way to achieve and map browsing function operation; inquiries and positioning tower information; information on the tower entry and editing.

\section{The Design Principles}

Security systems should be designed to prevent data theft and illegal landing and resources are hacking design through effective means. It involves information systems, data access and security of storage, data access control in strict accordance with its mandate, the data information is not lost, the user operates the data is in safe mode, and the operation content stream can be traced trail.

The system should be more than the concurrent operation and long-line system must ensure efficient service. To provide a good user interface, and give full consideration to the operating frequency, rational distribution, the system can work efficiently. It provides a good response speed, efficient support for business operations.

In the application of the system, under normal circumstances, due to the delay caused by the network and processing system without affecting the operation, other than not feel a significant delay operation means. Information is accurate, the data obtained from the interface with the actual basic data protection agreement, the attribute data reflect the actual situation, so as to provide the correct reference and help for the operation and maintenance work.

The system not only to meet the business requirements at this stage, but also to be able to meet future demand growth of business and development of new technologies to support future systems continuously updated and easy to upgrade. Host system should have good scalability to meet the requirements of different scale computing environments.

System requirements for using JAVA language, JAVA computer language is one for the development of the Internet, is a language that can be executed on any machine can be applications via the Internet on the local end of the transmission from the remote machine to the server and executed. Developed technology uses Ajax, is a collection of all current technologies in the browser via javaScript can be used. GIS application platform choose the open source and free GeoServer platform, using Geoserver the data as maps / images to publish, while taking advantage of the WFS-T can repair, delete, and new features. MS SQL Server database platform large database, all the map data and business data stored in the database.

\section{The Specific Functions}

Implement of GIS functions. In the hierarchical display of GIS geographic graphic interface, such as roads, rivers and obstacles, the layer can be pretreated, transmission line display for standard data collected by GPS positioning on the line, while GIS provides a graphic manipulation functions , graphics zoom, roam, and graphics distance, area calculations.

With the acceleration of urban and rural construction, urban rapid changes, general electronic map can only display in county roads, and cannot display the path to reach the next tower line, the line cannot meet the patrol line navigation role, in order to ensure the electronic map of timeliness, accuracy nature, electronic maps have basic ability to modify, can quickly and easily increase not marked on the map of roads, rivers and obstacles, so that GIS related information intuitive and effective, to provide the basis for the operation of the power system maintenance. 
Query function provides interactive GIS and DMS achieve queries, GIS display bar number, type and other basic information from the GIS database associated with real-time call lines, towers more information and related data records.

Implement of GPS Functions. In the mobile terminal, linesmen patrol uses a handheld GPS device, through inspection records waypoints and means to save the linesmen patrol inspection records. If the polling staff did not reach the point of inspection targeting, you cannot enter Record inspection records, GPS handset automatically records the time of the inspection tasks ,, as a basis for testing the effectiveness of the patrol inspection work.

At the end of the outdoor inspections, patrol lineman using a data cable to connect the handheld GPS with the computer, the software will patrol through the communication line and save data to the central database. Any terminal operator in the LAN, through different access rights, you can query the inspection staff working hours, appropriate equipment operating data, statistical check by security module can test the rate of inspection personnel inspection rate in place, the device, change of equipment operation indicators, timing and results processing equipment anomalies, statistical results can be printed out by means of various business reports.

Implement of DMS Functions. With the power companies increasing degree of information technology, various types of transmission lines appear information management systems that provide different levels of line resource management needs, and for the entry, without exception, are based on the information transmission line manual entry calculating, in the presence of time-consuming, obsolete data, and error-prone, cannot update other ills. The GPS positioning after editing already forming lines and line towers latitude and longitude data. Use latitude and longitude data, and tower type editor, can be convenient statistics of the number of each type of tower, span, length-resistant sheets, the distance from the line length value is particularly suitable for a series of substation line drawings insufficiency, due to the occurrence of technological transformation line change, full or partial resistance segments Zhang needs to recalculate the statistics line.

Some tower manual entry of information, such as insulators model, on cables and some other non-GPS acquisition parameters, to make computer statistics constitutes the foundation line database, greatly reducing the workload of manual entry and improve efficiency ,, because when the line when technological change, transformation and other reasons, as long as can be repositioned so that the data updated in real time after the implementation of standardized management of line maintenance content, can form a closed loop line maintenance in accordance with the content of entry to amend the relevant data so that the data, once data entry, the same data with the entry of changes and modifications to make the system scalability strong, extending the software life cycle.

\section{The Expansion of GIS Functions}

Development of GIS features only the first step, now there is no transmission GIS can accurately reflect the complete road map and route instructions at anytime, anywhere updated features, along with the computer technology matures, the next work area plans to update the GIS map as the focus of research while the GIS ported to PDA handset so even if there is no line of work experience, people can accurately reach the designated line tower, it has a wireless data transmission can make line maintenance, inspection can be done on-line monitoring and remote scheduling, do control, in control. Transmission Lines GIS emergency rescue management system based on the existing terminal, quick response and fault location fault tower, directing rescue personnel quickly arrived, so that transmission line to achieve integrated management requirements.

\section{References}

[1] Jia Xinzhang, 18570-2006 overhead transmission line inspection system, Vol. 6 (2004) No 53, p.25-26

[2] Peng Sue, Power distribution SCADA system integrated Information Technology Co., Ltd. Haijie lion, Vol. 12 (2005) No 27, p.74-76 
河南省 信阳市 浉河区 五星街道河南省信阳市宝石桥电力生产基地 4 楼 517464000 贾 阳 13849721660 0376-6218861 